\title{
Soft k-Uni Ideals of Semirings and its Algebraic Applications
}

Filiz ÇITAK ${ }^{1}$

ABSTRACT: In this paper, firstly, information about soft set introduced by Molodtsov is given. Then, the operations on the soft set are introduced. After then a definition of soft uni-k-ideal of a semiring by using the union operation of sets is given. Finally, some algebraic applications by using soft uni-k-ideal are investigated.

Keywords: Soft sets, k-ideals, soft uni-ideals, soft k-uni ideals.

\section{Yarıhalkaların Esnek k-Birleşimsel İdealleri ve Cebirsel Uygulamaları}

ÖZET: Bu çalışmada, ilk olarak Molodtsov tarafından ortaya atılan esnek küme teorisi hakkında bilgi verildi. Sonra esnek küme üzerindeki işlemler tanıtıldı. Daha sonra kümelerin birleşim işlemi kullanılarak bir yarıhalkanın esnek birleşimsel k-ideal tanımı verildi. Son olarak, esnek birleşimsel k-ideali kullanılarak bazı cebirsel uygulamalar araştırıldı.

Anahtar Kelimeler: Esnek kümeler, k-idealler, esnek birleşimsel idealler, esnek k-birleşimsel idealler

Filiz ÇITAK (0000-0003-1784-1845), Gaziosmanpaşa Üniversitesi, Fen Edebiyat Fakültesi, Matematik Bölümü, Tokat, Türkiye Sorumlu yazar/Corresponding Author: Filiz ÇITAK, filiz.citak@gop.edu.tr 


\section{INTRODUCTION}

Recently, soft set theory have been investigated intensively. Firstly, Molodtsov (Molodtsov, 1999) suggested soft set theory. Operations of soft sets are investigated by Maji et al. (Maji et al., 2003). Publications on soft set theory are continuing rapidly. Then, Çağman and Enginoğlu redefined definition of the soft set and investigated some properties (Çağman and Enginoğlu, 2010). Firstly, Aktaş and Çağman studied on soft algebra (Aktaş and Çağman, 2007). They defined a soft group and investigated its algebraic property. Acar et al. studied on the soft ring (Acar et al., 2010). Feng et al. defined the soft semiring and soft ideal (Feng et al., 2008). The concept of idealistic soft BCK/ BCI-algebras was introduced by Jun and Park (Jun and Park, 2008). Sun et al. studied the soft modules (Sun et al., 2008). Çağman et al. studied the concept of soft int-groups and investigated algebraic properties of soft int-group (Çağman et al., 2012). Then Çıtak and Çağman gave a definition of soft int-ring (Çıtak and Çağman, 2015). Sezgin et al. expressed a definition of soft uni-group and investigated some properties of soft uni-group (Sezgin et al., 2015). Soft BL-algebras are introduced by Zhan and Jun (Zhan and Jun, 2010).

Then, Zhan and $\mathrm{Xu}$ introduced the soft lattice implication algebras (Zhan and Xu,2011). Atagün and Sezgin studied the int-soft substructures of groups and semirings (Atagün and Sezgin, 2017). In recent years, soft algebraic structures have been studied quickly (Ali et al. 2009, Ali et al. 2011, Lee et al. 2014, Sezgin et al. 2014, Jana and Pal 2016, Sezgin 2017, Ma et al. 2017).

In this paper, we define the concept of soft uni-ideal of a semiring. We also introduce the concept of soft k-uni-ideal of a semiring by using intersection operation of the set and investigate the basic properties of soft k-uni-ideal. Moreover, we define a soft k-product of two soft left k-uni-ideals and works on their algebraic structures in detail.

\section{MATERIALS AND METHODS}

Throughout this work, $U$ is a universal set, $E$ is a set of parameters, $X \subseteq E$ and $P(U)$ is the power set of $U$.

Definition 1. A nonempty set $S$ together with a binary operation $*$ is a semigroup if $*$ is associative in $S$, that is, for all $a, b, c \in S, a *(b * c)=(a * b) * c$.

A semigroup is commutative, if $*$ is commutative in $S$, that is, for all $a, b \in S$, 
$a * b=b * a$

(Golan, 1992).

Definition 2. A semiring is a nonempty set $S$ together with two binary operations addition and multiplication denoted by “+ ", “.” respectively, satisfying

i. $(S,+)$ is a commutative semigroup,

ii. $(S, \cdot)$ is a semigroup,

iii. distributive low holds, that is, for all $a, b, c \in S, a \cdot(b+c)=a \cdot b+a \cdot c$ and $(a+b) \cdot c=a \cdot c+b \cdot c$

(Golan, 1992).

Henceforth, $S$ and $R$ is a semiring.

Definition 3. A subset $I$ of $S$ is a left (right) ideal of $S$, if

i. $\quad a+b \in I$ for all $a, b \in I$

ii. $\quad b \cdot a \in I(a \cdot b \in I)$ for any $a \in I$ and $b \in S$

If $I$ is both a left and right ideal, then $I$ is an ideal (Golan, 1992).

Definition 4. Let $I$ be a left (right) ideal of S. A left (right) ideal of $S$ is a left (right) k-ideal of $S$ if $b, c \in I, a \in S, a+b=c$ implies $a \in I$ (La Torre, 1965).

Definition 5. $F_{X}$ is a soft set over $U$ where $F_{X}: E \rightarrow P(U)$ is a function such that $F_{X}(a)=\varnothing$ if $a \notin X$ (Molodtsov, 1999).

The set of soft sets is symbolized by $S(U)$.

Definition 6. Let $F_{X} \in S(U) . F_{X}$ is an empty soft set if $F_{X}(a)=\varnothing$ for each $a \in X$. An 
empty soft set is symbolized by $\tilde{\varnothing}$.

$F_{X}$ is said to be a universal soft set if $F_{X}(a)=U$ for each $a \in X$. A universal soft set is symbolized by $\tilde{A}$ (Maji et al., 2003).

Definition 7. Let $F_{X}, G_{X} \in S(U) . F_{X}$ is said to be a soft subset of $G_{X}$, if $F_{X}(a) \subseteq G_{X}(a)$ for each $a \in X$. A soft subset is symbolized by $F_{X} \subseteq G_{X}$.

$F_{X}$ and $G_{X}$ said to equal soft sets if $F_{X}(a)=G_{X}(a)$ for each $a \in X$. Equal soft sets are symbolized by $F_{X} \cong G_{X}$ (Çağman and Enginoğlu, 2010).

Definition 8. Let $F_{X}, G_{X} \in S(U)$. The union of $F_{X}$ and $G_{X}$ is defined by $\left(F_{X} \cup G_{X}\right)(a)=F_{X}(a) \cup G_{X}(a)$ for each $a \in X$.

The intersection of $F_{X}$ and $G_{X}$ is defined by $\left(F_{X} \cap G_{X}\right)(a)=F_{X}(a) \cap G_{X}(a)$ for each $a \in X$. They are symbolized by $F_{X} \tilde{\cup} G_{X}$ and $F_{X} \tilde{\cap} G_{X}$, respectively (Çağman and Enginoğlu, 2010).

Definition 9. Let $F_{X}, G_{X} \in S(U) . \wedge$-product and $\vee$-product of $F_{X}$ and $G_{X}$ are defined by $\left(F_{X} \wedge G_{X}\right)(a, b)=F_{X}(a) \cap G_{X}(b)$ and $\left(F_{X} \vee G_{X}\right)(a, b)=F_{X}(a) \cup G_{X}(b)$ for each $a, b \in X$, respectively. They are symbolized by $F_{X} \wedge G_{X}$ and $F_{X} \vee G_{X}$, respectively (Çağman and Enginoğlu, 2010).

Definition 10. Let $F_{X} \in S(U)$ and $\varphi$ be a function from a set $X$ to a set $Y$. For all $b \in Y, \varphi\left(F_{X}\right): Y \rightarrow P(U), \varphi\left(F_{X}\right)(b)=\left\{\begin{array}{cl}\cup\left\{F_{X}(a): a \in X, \varphi(a)=b\right\} & \text { if } b \in \varphi(X) \\ \varnothing, & \text { if } b \notin \varphi(X)\end{array}\right.$ is a soft image of $F_{X}$ under $\varphi$. For all $a \in X$, 
$\varphi^{-1}\left(F_{X}\right): X \rightarrow P(U), \varphi^{-1}\left(F_{X}\right)(a)=F_{X}(\varphi(a))$ is a soft preimage (or soft inverse image) of $F_{X}$ under $\varphi$ (Çağman et al., 2012).

Definition 11. Let $F_{X} \in S(U)$ and $A \in P(U)$. A set $F_{X}^{\subseteq A}=\left\{a \in X: F_{X}(a) \subseteq A\right\}$ is a lower $A$-inclusion of $F_{X}$ (Sezgin et al., 2014).

Definition 12. The relative complement of the soft set $F_{X}$ over $\mathrm{U}$ is denoted by $F_{X}^{r}$, where $F_{X}^{r}: X \rightarrow P(U)$ is a mapping given as $F_{X}^{r}(a)=U-F_{X}(a)$ for all $a \in X$ (Sezgin et al., 2014).

Definition 13. Let $S$ be a ring and $F_{S} \in S(U) . F_{S}$ is called a soft left (right) int-ideal over $U$, if it satisfies the following axioms:

$$
\begin{array}{ll}
\text { i. } & F_{S}(a+b) \supseteq F_{S}(a) \cap F_{S}(b) \\
\text { ii. } & F_{S}(a b) \supseteq F_{S}(b)\left(F_{S}(a b) \supseteq F_{S}(a)\right)
\end{array}
$$

for all $a, b \in S$.

$F_{S}$ is a soft int-ideal over $U$, if it is both soft left int-ideal and soft right int-ideal over $U$ (Çıtak and Çağman, 2015).

Definition 14. Let $S$ be a semiring and $F_{S} \in S(U) . F_{S}$ is called a soft left (right) intideal over $U$, if it satisfies the following axioms:

(i) $F_{S}(a+b) \supseteq F_{S}(a) \cap F_{S}(b)$

(ii) $F_{S}(a b) \supseteq F_{S}(b)\left(F_{S}(a b) \supseteq F_{S}(a)\right)$

for all $a, b \in S$.

$F_{S}$ is a soft int-ideal over $U$, if it is both soft left int-ideal and soft right int-ideal over $U$ (Çıtak, 2017). 


\section{RESULTS AND DISCUSSION}

In this section, we first introduce a concept of soft uni-ideal of a semiring. Then, we give the concept of soft k-uni-ideal of a semiring by using union operation of set and investigate the basic properties of soft k-uni-ideal. Finally, we define a soft k-product of two soft left k-uni-ideals and study their algebraic structures.

Definition 1. Let $F_{S} \in S(U) . F_{S}$ is called a soft left (right) uni-ideal over $U$, if it satisfies the following axioms:

(i) $F_{S}(a+b) \subseteq F_{S}(a) \cup F_{S}(b)$

(ii) $F_{S}(a b) \subseteq F_{S}(b)\left(F_{S}(a b) \subseteq F_{S}(a)\right)$

for all $a, b \in S$.

$F_{S}$ is a soft uni-ideal over $U$, if it is both soft left uni-ideal and soft right uni-ideal over $U$.

Definition 2. Let $F_{S}$ be a soft left uni-ideal over $U . F_{S}$ is called a soft left k-uni-ideal over $U$, if for each $a, b, c \in S, a+b=c$ implies $F_{S}(a) \subseteq F_{S}(b) \cup F_{S}(c)$.

A soft right k-uni-ideal can be defined similarly.

Theorem 3. Let $F_{S}$ and $G_{S}$ be two soft left (right) k-uni-ideals over $U$. Then, $F_{S} \cup \tilde{U} G_{S}$ is a soft left (right) k-uni-ideal over $U$. a soft left (right) k-uni-ideal over $U$. 
Proof. Let $a, b, c \in S$ such that $a+b=c$. Then,

$$
\begin{aligned}
\left(F_{S} \cup G_{S}\right)(a) & =F_{S}(a) \cup G_{S}(a) \\
& \subseteq F_{S}(b) \cup F_{S}(c) \cup G_{S}(b) \cup G_{S}(c) \\
& =F_{S}(b) \cup G_{S}(b) \cup F_{S}(c) \cup G_{S}(c) \\
& =\left(F_{S} \cup G_{S}\right)(b) \cup\left(F_{S} \cup G_{S}\right)(c)
\end{aligned}
$$

Therefore, $F_{S} \tilde{\cup} G_{S}$ is a soft left k-uni-ideal over $U$.

Remark 4. The following example shows that $F_{S} \tilde{\cap} G_{S}$ is not a soft k-uni-ideal over $U$.

Example 5. Assume that set of the naturel numbers $\mathbb{N}$ is the universal set and $S=x, y, z$ is the subset of set of parameters. The soft k-uni-ideals $F_{S}$ and $G_{S}$ over $\mathbb{N}$ are defined as

and

\begin{tabular}{l|l|l|l}
+ & $\mathrm{x}$ & $\mathrm{y}$ & $\mathrm{z}$ \\
\hline $\mathrm{x}$ & $\mathrm{x}$ & $\mathrm{y}$ & $\mathrm{y}$ \\
\hline $\mathrm{y}$ & $\mathrm{y}$ & $\mathrm{x}$ & $\mathrm{x}$ \\
\hline $\mathrm{z}$ & $\mathrm{y}$ & $\mathrm{x}$ & $\mathrm{x}$
\end{tabular}

\begin{tabular}{l|l|l|l}
$\cdot$ & $\mathrm{x}$ & $\mathrm{y}$ & $\mathrm{z}$ \\
\hline $\mathrm{x}$ & $\mathrm{z}$ & $\mathrm{z}$ & $\mathrm{z}$ \\
\hline $\mathrm{y}$ & $\mathrm{z}$ & $\mathrm{z}$ & $\mathrm{z}$ \\
\hline $\mathrm{z}$ & $\mathrm{z}$ & $\mathrm{z}$ & $\mathrm{z}$
\end{tabular}

$$
\begin{aligned}
& F_{S}(x)=\{2,4,6\}, F_{S}(y)=\{1,2,3,4,5,6,8,18\}, F_{S}(z)=\{2,4,6\} \text { and } G_{S}(x)=\{2,12,22\}, \\
& G_{S}(y)=\{2,8,12,18,22\}, G_{S}(z)=\{2,12,22\} .
\end{aligned}
$$


It shows that $\left(F_{S} \cap G_{S}\right)(x+z) \not \subset\left(F_{S} \cap G_{S}\right)(x) \cup\left(F_{S} \cap G_{S}\right)(z)$. Then, $F_{S} \tilde{\cap} G_{S}$ is not a soft uni-ideal over $\mathbb{N}$. Therefore, $F_{S} \tilde{\cap} G_{S}$ is not a soft k-uni-ideal over $\mathbb{N}$.

Theorem 6. Let $F_{S}$ be a soft left (right) k-uni-ideal over $U$ and $G_{R}$ be a soft left (right) k-uni-ideal over $U$. Then, $F_{S} \vee G_{R}$ is a soft left (right) k-uni-ideal over $U$.

Proof. Let $\left(a_{1}, a_{2}\right),\left(b_{1}, b_{2}\right),\left(c_{1}, c_{2}\right) \in S \times R$ such that $\left(a_{1}, a_{2}\right)+\left(b_{1}, b_{2}\right)=\left(c_{1}, c_{2}\right)$. Hence,

$$
\begin{aligned}
\left(F_{S} \vee G_{R}\right)\left(a_{1}, a_{2}\right) & =F_{S}\left(a_{1}\right) \cup G_{R}\left(a_{2}\right) \\
& \subseteq F_{S}\left(b_{1}\right) \cup F_{S}\left(c_{1}\right) \cup G_{R}\left(b_{2}\right) \cup G_{R}\left(c_{2}\right) \\
& =F_{S}\left(b_{1}\right) \cup G_{R}\left(b_{2}\right) \cup F_{S}\left(c_{1}\right) \cup G_{R}\left(c_{2}\right) \\
& =\left(F_{S} \cup G_{R}\right)\left(b_{1}, b_{2}\right) \cup\left(F_{S} \cup G_{R}\right)\left(c_{1}, c_{2}\right)
\end{aligned}
$$

Therefore, $F_{S} \vee G_{R}$ is a soft left k-uni-ideal over $U$.

Remark 7.The following example shows that $F_{S} \wedge G_{R}$ is not a soft k-uni-ideal over $U$.

Example 8. Consider $F_{S}$ in Example 3.6. Also let $G_{\mathbb{Z}_{4}}$ be a soft k-uni-ideal over $\mathbb{N}$ defined by $\quad G_{\mathbb{Z}_{4}}(0)=\{3,5\}, \quad G_{\mathbb{Z}_{4}}(1)=\{1,3,5,7,9\}, \quad G_{\mathbb{Z}_{4}}(2)=\{1,3,5,7\}$, $G_{\mathbb{Z}_{4}}(3)=\{1,3,5,7,9\}$

It shows that $\left(F_{S} \wedge G_{\mathbb{Z}_{4}}\right)[(x, 2)+(z, 3)] \not \subset\left(F_{S} \wedge G_{\mathbb{Z}_{4}}\right)(x, 2) \cup\left(F_{S} \cap G_{\mathbb{Z}_{4}}\right)(z, 3)$. Then, $F_{S} \wedge G_{\mathbb{Z}_{4}}$ is not a soft uni-ideal over $\mathbb{N}$. Therefore, $F_{S} \wedge G_{\mathbb{Z}_{4}}$ is not a soft k-uni-ideal over $\mathbb{N}$.

Definition 9. Let $F_{S}$ and $G_{S}$ be two soft sets over $U$. Then, $F_{S} \tilde{\odot}_{k} G_{S}$ is called a soft kuni-product where

$$
\left(F_{S} \tilde{\odot}_{k} G_{S}\right)(a)=\left\{\begin{array}{cc}
\cup F_{S}\left(a_{i}\right) \cup G_{S}\left(b_{i}\right): i=1,2, & \text { if } a+a_{1} b_{1}=a_{2} b_{2} \\
\varnothing & \text { if a cannot be predicated as } a+a_{1} b_{1}=a_{2} b_{2}
\end{array}\right.
$$


Theorem 10 Let $F_{S}$ be a soft right k-uni-ideal and $G_{S}$ be a soft left k-uni-ideal over $U$.

Then, $F_{S} \tilde{\bigodot}_{k} G_{S} \supseteq F_{S} \tilde{\cup} G_{S}$.

Proof. Let $a \in S$. If $\left(F_{S} \widetilde{\odot}_{k} G_{S}\right)(a)=\varnothing$, then it is a clear proof. Let $\left(F_{S} \tilde{\odot}_{k} G_{S}\right)(a) \neq \varnothing$ . Since $F_{S}$ is a soft right k-uni-ideal over $U$, then

$$
\begin{aligned}
F_{S}(a) & \subseteq F_{S}\left(a_{1} b_{1}\right) \cup F_{S}\left(a_{2} b_{2}\right) \\
& \subseteq F_{S}\left(a_{1}\right) \cup F_{S}\left(a_{2}\right)
\end{aligned}
$$

for all $a_{i}, b_{i} \in S, i=1,2$, satisfying $a+a_{1} b_{1}=a_{2} b_{2}$. Similarly

$$
G_{S}(a) \subseteq G_{S}\left(b_{1}\right) \cup G_{S}\left(b_{2}\right)
$$

Thus,

$$
\begin{aligned}
\left(F_{S} \odot_{k} G_{S}\right)(a) & =\cup F_{S}\left(a_{i}\right) \cup G_{S}\left(b_{i}\right): i=1,2, a+a_{1} b_{1}=a_{2} b_{2} \\
& \supseteq F_{S}(a) \cup G_{S}(a) \\
& =\left(F_{S} \cup G_{S}\right)(a)
\end{aligned}
$$

Therefore, $F_{S} \tilde{\bigodot}_{k} G_{S} \supseteq F_{S} \tilde{\cup} G_{S}$.

Lemma 11. Let $F_{S} \in S(U) . F_{S}$ is a soft left (right) uni-ideal over $U$ iff $F_{S}^{\subseteq A}$ is a left (right) ideal of $S$, for any $A \in P(U)$ such that $F_{S}^{\subseteq A} \neq \varnothing$.

Proof. Assume that $F_{S}$ is a soft left uni-ideal over $U$. Let $a, b \in F_{S}^{\subseteq A}$. Then, $F_{S}(a) \subseteq A$ and $F_{S}(b) \subseteq A$. It follows that

$$
\begin{aligned}
F_{S}(a+b) & \subseteq F_{S}(a) \cup F_{S}(b) \\
& \subseteq A
\end{aligned}
$$

Thus, $a+b \in F_{S}^{\subseteq A}$. Let $a \in F_{S}^{\subseteq A}$ and $s \in S$. It shows that $F_{S}(a) \subseteq A$. And then, 


$$
\begin{aligned}
F_{S}(s a) & \subseteq F_{S}(a) \\
& \subseteq A
\end{aligned}
$$

Thus, $s a \in F_{S}^{\subseteq A}$. Therefore, $F_{S}^{\subseteq A}$ is a left ideal of $S$, for every $A \in P(U)$ such that $F_{S}^{\subseteq A} \neq \varnothing$.

Conversely, let $F_{S}^{\subseteq A}$ be a left ideal of $S$, for every $A \in P(U)$ such that $F_{S}^{\subseteq A} \neq \varnothing . \quad a, b \in F_{S}^{\subseteq B} \quad$ such that $B=F_{S}(a) \cup F_{S}(b)$ for each $a, b \in S$. Hence, $a+b \in F_{S}^{\subseteq B}$. Thus,

$$
\begin{aligned}
F_{S}(a+b) & \subseteq B \\
& =F_{S}(a) \cup F_{S}(b)
\end{aligned}
$$

Also, $a \in F_{S}^{\subseteq C}$ such that $C=F_{S}(a)$, we obtain $s a \in F_{S}^{\subseteq C}$ for each $s \in S$. Then, $F_{S}(s a) \subseteq F_{S}(a)$. Therefore, $F_{S}$ is a soft left uni-ideal over $U$.

Theorem 12. Let $F_{S} \in S(U) . F_{S}$ is a soft left (right) k-uni-ideal over $U$ iff $F_{S}^{\subseteq A}$ is a left (right) k-ideal of $S$ for any $A \in P(U)$ such that $F_{S}^{\subseteq A} \neq \varnothing$.

Proof. Following Lemma 11, it was proved that a soft set $F_{S}$ is a soft left uni-ideal iff $G_{S}^{\subseteq A}$ is a left ideal of $S$ for any $A \in P(U)$ such that $F_{S}^{\subseteq A} \neq \varnothing$. Suppose that $F_{S}$ is a soft left k-uni ideal over $U$. Let $a, k \in F_{S}^{\subseteq A}, s \in S, s+a=k$. Since $a, k \in F_{S}^{\subseteq A}$, we have $F_{S}(a) \subseteq A, F_{S}(k) \subseteq A$. Also, $F_{S}(s) \subseteq F_{S}(a) \cup F_{S}(k)$. Thus, $F_{S}(s) \subseteq A$, and so $s \in F_{S}^{\subseteq A}$. Hence, $F_{S}^{\subseteq A}$ is a left k-ideal of $S$.

Conversely, let $F_{S}^{\subseteq A}$ be a left k-ideal of $S$, for any $A \in P(U)$ such that $F_{S}^{\subseteq A} \neq \varnothing$. Let $a, s, k \in S \quad$ such that $a+s=k$. Suppose that $F_{S}(s)=A_{1}, F_{S}(k)=A_{2}\left(A_{i} \in P(U)\right)$. Let $A_{1} \cup A_{2}=A$. Then, $s \in F_{S}^{\subseteq A}$ and $k \in F_{S}^{\subseteq A}$. 
Since $F_{S}^{\subseteq A}$ is a left k-ideal of $S$, we have $a \in F_{S}^{\subseteq A}$, i.e. $F_{S}(a) \subseteq F_{S}(s) \cup F_{S}(k)$. Thus, $F_{S}$ is a soft left k-uni-ideal over $U$.

Lemma 13. Let $F_{S} \in S(U) . F_{S}$ is a soft left (right) uni-ideal set over $U$ iff $F_{S}^{r}$ is a left (right) int-ideal over $U$.

Proof. Let $F_{S}$ is a soft left (right) uni-ideal over $U$. Then,

$$
\begin{aligned}
F_{S}^{r}(a+b) & =U-F_{S}(a+b) & & F_{S}^{r}(a b) \\
& \supseteq U-F_{S}(a) \cup F_{S}(b) & \text { and } & \supseteq U-F_{S}(a b) \\
& =U-F_{S}(a) \cap U-F_{S}(b) & & =F_{S}^{r}(a) \\
& =F_{S}^{r}(a) \cap F_{S}^{r}(b) & &
\end{aligned}
$$

Therefore, $F_{S}^{r}$ is a soft left int-ideal over $U$.

Conversely, let $F_{S}^{r}$ be a soft left (right) int-ideal over $U$. Then,

$$
\begin{aligned}
F_{S}(a+b) & =U-F_{S}^{r}(a+b) \\
& \subseteq U-\left[F_{S}^{r}(a) \cap F_{S}^{r}(b)\right] \\
& =\left[U-F_{S}^{r}(a)\right] \cup\left[U-F_{S}^{r}(b)\right] \\
& =F_{S}(a) \cup F_{S}(b)
\end{aligned}
$$

$$
\text { and } \quad \begin{aligned}
F_{S}(a b) & =U-F_{S}^{r}(a b) \\
& \subseteq U-F_{S}^{r}(a) \\
& =F_{S}(a)
\end{aligned}
$$

Therefore, $F_{S}$ is a soft left uni-ideal over $U$.

Theorem 14. Let $F_{S} \in S(U) . F_{S}$ is a soft left (right) k-uni-ideal over $U$ iff $F_{S}^{r}$ is a left (right) k-int-ideal over $U$.

Proof. By Lemma 13, we know that $F_{S}$ is a soft left (right) uni-ideal over $U$ iff $F_{S}^{r}$ is a left (right) int-ideal over $U$.

Let $F_{S}$ is a soft left (right) k-uni-ideal over $U$. Let $a, b, c \in S$ such that $a+b=c$. Then, 


$$
\begin{aligned}
F_{S}^{r}(a) & =U-F_{S}(a) \\
& \supseteq U-F_{S}(b) \cup F_{S}(c) \\
& =U-F_{S}(b) \cap U-F_{S}(c) \\
& =F_{S}^{r}(b) \cap F_{S}^{r}(c)
\end{aligned}
$$

Therefore, $F_{S}^{r}$ is a soft left k-int-ideal over $U$.

Conversely, let $F_{S}^{r}$ is a soft left (right) k-int-ideal over $U$. Let $a, b, c \in S$ such that $a+b=c$. Then,

$$
\begin{aligned}
F_{S}(a) & =U-F_{S}^{r}(a) \\
& \subseteq U-\left[F_{S}^{r}(b) \cap F_{S}^{r}(c)\right] \\
& =\left[U-F_{S}^{r}(b)\right] \cup\left[U-F_{S}^{r}(c)\right] \\
& =F_{S}(b) \cup F_{S}(c)
\end{aligned}
$$

Therefore, $F_{S}$ is a soft left k-uni-ideal over $U$.

Theorem 15. Let $\varphi$ be a surjective homomorphism from $R$ to $S$. Let $F_{R}$ be a soft left (right) k-uni-ideal over $U \cdot \varphi^{*}\left(F_{R}\right)_{S}$ is a soft left (right) k-uni-ideal over $U$.

Proof. Let $F_{R}$ be a soft left (right) k-uni-ideal over $U$. By Theorem 3.14, $F_{R}^{r}$ is a soft left (right) k-int-ideal over $U$. By (Sezgin et al., 2015), $\varphi\left(F_{R}^{r}\right)_{S}$ is a soft left (right) kint-ideal over $U \cdot\left(\varphi^{*}\left(F_{R}\right)_{S}\right)^{r}$ is a soft left (right) k-int-ideal over $U$ since $\varphi\left(F_{R}^{r}\right)_{S}=\left(\varphi^{*}\left(F_{R}\right)_{S}\right)^{r}$ by (Sezgin et al., 2015). If $\left(\varphi^{*}\left(F_{R}\right)_{S}\right)^{r}$ is a soft left (right) k-intideal over $U$, then $\varphi^{*}\left(F_{R}\right)_{S}$ is a soft left (right) k-uni-ideal over $U$.

Theorem 16. Let $\varphi$ be a surjective homomorphism from $R$ to $S$. Let $F_{S}$ be a soft left (right) k-uni-ideal over $U \cdot \varphi^{-1}\left(F_{S}\right)_{R}$ is a soft left(right) k-uni-ideal over $U$. 
Proof. For any $a, b, c \in S$ such that $a+b=c(\varphi(a)+\varphi(b)=\varphi(c))$,

$$
\begin{aligned}
\varphi^{-1}\left(F_{S}\right)(a) & =F_{S}(\varphi(a)) \\
& \subseteq F_{S}(\varphi(b)) \cup F_{S}(\varphi(c)) \\
& =\varphi^{-1}\left(F_{S}\right)(b) \cup \varphi^{-1}\left(F_{S}\right)(c)
\end{aligned}
$$

proving that $\varphi^{-1}\left(F_{S}\right)_{R}$ is a soft left (right) k-uni-ideal over $U$.

\section{CONCLUSION}

Soft k-uni-ideal and their algebraic properties were reseached by using soft sets and union of sets in this paper. In the following studies, soft h-uni-ideal of a hemiring and their algebraic properties can reseached by a reseacher.

\section{REFERENCES}

Acar U, Koyuncu F and Tanay B, 2010. Soft Sets and Soft Rings, Computers and Mathematics with Applications, 59: 3458-3463.

Aktaş H and Çağman N, 2007. Soft Sets and Soft Groups, Information Sciences, 177: 2726-2735.

Ali MI, Feng F, Liu X, Min WK and Shabir M, 2009. On Some New Operations in Soft Set Theory, Computers and Mathematics with Application, 57: 1547-1553.

Ali MI, Shabir M and Naz M, 2011. Algebraic Structures of Soft Sets Associated with New Operations, Computers and Mathematics with Application, 61: 2647-2654.

Atagün AO and Sezgin A, 2017. Int-Soft Substructures of Groups and Semirings with Applications, Applied Mathematics \& Information Sciences, 11 (1): 105-113.

Çağman N and Enginoğlu S, 2010. Soft Set Theory and uniint Decision Making, European Journal of Operational Research, 207: 848-855.

Çağman N, Çıtak F and Aktaş H, 2012. Soft int-group and its Applications to Group Theory, Neural Computing and Applications, 21: 151-158.

Çıtak F and Çağman N, 2015. Soft int-rings and its Algebraic Applications, Journal of Intelligent and Fuzzy Systems, 28: $1225-1233$
Feng F, Jun YB and Zhao X, 2008. Soft Semirings, Computers and Mathematics with Applications, 56: 2621-2628.

Golan J S, 1992. The Theory of Semirings with Applications in Mathematics and Theoritical Computer Science, Longman Scientific and Technical, U.K.

Jana C and Pal M, 2016. Applications of New Soft Intersection Set on Groups, Annals of Fuzzy Mathematics and Informatics, 11 (6): 923-944.

Jun YB and Park CH, 2008. Applications of Soft Sets in Ideal Theory of BCK/BCI-Algebras, Information Sciences, 178: 2466-2475.

La Torre DR, 1965. On h-Ideals and k-Ideals in Hemirings, Publicationes Mathematicae Debrecen, 12: 219-226.

Lee JH, Kong IS, Kim HS and Jung JU, 2014. Generalized intSoft Subsemigroups, Annals of Fuzzy Mathematics and Informatics, 8 (6): 869-887.

Ma X, Zhan J and Ali MI, 2017. Applications of a Kind of Novel Z-Soft Fuzzy Rough Ideals to Hemirings, Journal of Intelligent and Fuzzy Systems, 32 (3) 1-12.

Maji PK, Biswas R and Roy AR, 2003. Soft Set Theory, Computers and Mathematics with Applications, 45: 555562. 
Molodtsov DA, 1999. Soft Set Theory-First Results, Computers and Mathematics with Applications, 37: 19-31.

Sezgin A, Çağman N and Atagün AO, 2015. Uni-Soft Substructures of Groups, Annals of Fuzzy Mathematics and Informatics, 9 (2): 235-246.

Sezgin A, 2017. Characterizations of Certain Classes of Semigroups via Soft Intersection Ideals, Italian Journal of Pure and Applied Mathematics, 38(1).
Sun Q M, Zhang ZL and Liu J, 2008. Soft Sets and Soft Modules, Lecture Notes in Computer Science, 5009: 403-409.

Zhan J and Jun YB, 2010. Soft BL-Algebras Based on Fuzzy Sets, Computers and Mathematics with Applications, 59 (6): 2037-2046.

Zhan J and Xu Y, 2011. Soft Lattice Implication Algebras Based on Fuzzy Sets, Hacettepe Journal of Mathematics and Statistics, 40 (4): 483-492. 